\title{
IMPACT OF T WAVE AMPLITUDE IN LEAD aVR ON PREDICTING APPROPRIA- TE THERAPIES IN HYPERTROPHIC CARDIOMYOPATHY PATIENTS WITH AN IMPLANTABLE CARDIOVERTER DEFIBRILLATOR
}

\author{
Begüm Söyleyici ${ }^{1}$, Pelinsu Elif Hünkar ${ }^{1}$, Çağrı Girit ${ }^{1}$, Cansu Kurt ${ }^{1}$, Fatih Mehmet Uçar ${ }^{2}$ \\ ${ }^{1}$ Trakya University School of Medicine, Edirne, TURKEY \\ ${ }^{2}$ Department of Cardiology, Trakya University School of Medicine, Edirne, TURKEY
}

\section{ABSTRACT}

Aims: Although implantable cardioverter defibrillator reduces mortality in hypertrophic cardiomyopathy patients, inappropriate implantable cardioverter defibrillator shocks are related to increased mortality. The aim of this study is to investigate whether a new electrocardiographic marker of T wave amplitude in lead aVR can be used to predict appropriate therapy of implantable cardioverter defibrillator (shock or anti-tachycardia pacing) in hypertrophic cardiomyopathy patients.

Methods: Thirty-six hypertrophic cardiomyopathy patients, who were admitted to the outpatient clinic for pacemaker control, with implantable cardioverter defibrillator were retrospectively examined (mean age: $51 \pm 10.2$ years, $72.2 \%$ male). The primary endpoint was appropriate implantable cardioverter defibrillator therapy. All hematological, biochemical and electrocardiogram parameters were measured before implantable cardioverter defibrillator was implanted.

Results: Over a median follow-up period of 33 months, 9 (25\%) patients experienced appropriate implantable cardioverter defibrillator therapy. Heart rate and QRS interval were similar between groups. QT and QTc values were higher in patients that received appropriate shocks. Patients who have T wave inversion were higher in therapy positive group. T wave amplitude in lead aVR values were significantly associated with appropriate therapy.

Conclusion: Using simple ECG parameters, we may predict arrhythmic episodes before ICD implantation and an improvement of the medical antiarrhythmic therapy might be protective for HCM patients with ICD.

Keywords: Hypertrophic cardiomyopathy, defibrillator, electrocardiography

\section{INTRODUCTION}

Hypertrophic cardiomyopathy (HCM) is the most frequent hereditary cardiovascular disease in general population and seen in 1 in 500 equally on both sexes (1). It is characterized by increased ventricular wall thickness. HCM is associated with increased risk of atrial fibrillation, cardiac insufficiency and myocardial ischemia (2). Clinical manifestation of HCM might be shortness of breath, tachycardia, syncope, ischemia or abnormal vascularity. It is the prior cause of sudden death at early ages, with a $0.5-1 \%$ occurrence per year and $2.8 \%$ mortality rate in the follow-up (3-5).
Implantable cardioverter defibrillator (ICD) is a device which is implanted inside the body in order to provide cardioversion, defibrillation and pacing of the heart. ICD is remarkably effective in reducing the rate of sudden cardiac death due to ventricular tachyarrhythmia. It is used for several indications such as HCM, ischemic-nonischemic cardiomyopathy, Brugada syndrome and Long QT syndrome (6).

Nevertheless, inappropriate ICD therapy, which is caused by a failure of differentiating between supraventricular and ventricular tachyarrhythmia or noncardiac oversensing (e.g. myopotentials), has possibly life-threatening side effects. Inappropriate ICD therapy 
(inappropriate anti-tachycardia pacing and inappropriate shocks) or electrode displacement and dysfunction have been associated with myocardial damage and increased mortality. Additionally, inappropriate ICD therapy, especially inappropriate shock therapy, has been related to psychological distress and reduced quality of patients' life (6-8). Therefore, evaluating the adequacy of ICD therapy is highly important for patients. Besides, the association between TAaVR (T wave amplitude in lead aVR) and proper therapy of ICD in HCM patients is not known.

The aim of this study is to reveal the association between a simple electrocardiogram (ECG) parameter, $\mathrm{T}$ wave amplitude, on aVR derivation and appropriate ICD therapy (shock or anti-tachycardia pacing) in HCM patients.

\section{MATERIAL AND METHODS}

This study was approved by Scientific Research Ethics Committee of Trakya University Medical Faculty. The study is carried out according to the World Health Organization Helsinki Declaration and international ethical standards. Informed consent was obtained from all of the subjects. Patients over 18 years old who are diagnosed with hypertrophic cardiomyopathy, have implantable cardioverter defibrillator and were hospitalized in the cardiology clinic of Trakya University Hospital in between January 2017- 2018 constitute the universe of this retrospective research. 36 subjects were included in the study. Electrocardiograms and discharge reports of these patients are collected from the archive of Trakya University Faculty of Medicine through patients' medical record numbers. $T$ wave amplitudes in the aVR lead of the selected patients' electrocardiograms are examined for the most suitable ICD treatment. All of the measurements were made by the same cardiologist in order to standardize the results.

The 12-lead ECG was recorded at the $50 \mathrm{~mm} / \mathrm{s}$ speed (Nihon Kohden, Tokyo, Japan) in the supine position and at rest. During the evaluation of the patients, resting heart rate was measured from the ECG. To minimize the incorrect measurements, QT and QRS intervals were measured manually with calipers and magnifying glass. ECG measurements of QT and QRS intervals were done by two cardiologists who were blinded to the patient data. Measurement was done in lead II and lead V5 and the longest QT interval was chosen for the analysis. The QT interval was measured from the beginning of the QRS complex to the end of the T wave and was corrected using the Bazett's formula. $\mathrm{T}$ wave inversion was defined as a negative $\mathrm{T}$ wave ( $\mathrm{T}$ amp $<0 \mathrm{mV}$ ) in at least two contiguous leads except for aVR, V1, and V2 (9). The $\mathrm{T}$ wave amplitude was defined as the maximum deviation from the PR isoelectric baseline and/or the first deflection after the QRS complex (10).

The patients were divided into two groups regarding having an appropriate ICD therapy or not. The first group consisted of 27 patients, who had not recieved ICD therapy; while the second group included 9 patients.

After data collection, all of the data was analyzed by using SPSS software version 17.0. A p-value of $<0.05$ was evaluated as statistically significant. Continuous variables included median with interquartile range or mean \pm standard deviation of the data and categorical variables included numbers and percentages of the data. Chi-square test or Fisher's exact test was performed to associate the categorical variables. Data were analysed for normal dissemination using Mann-Whitney U test, Kolmogorov-Smirnov test or Student's t-test and was used for continuous variables, when appropriate. Pearson's correlation test was used for correlational analysis.

\section{RESULTS}

The mean age of the subjects was $51 \pm 10.2$ years; baseline clinical characteristics and laboratory parameters of the patients are shown in Table 1 . The baseline clinical and demographic characteristics of the patients were similar between groups ( $\mathrm{p}>0.05)$.

The ECG and echocardiographic results are shown in Table 2. There was no contrast between the two groups with regard to left ventricular ejection fraction $(\mathrm{p}=0.22)$ and left ventricular diameters $(\mathrm{p}=0.44$ and 0.36 , respectively). QTs interval and heart rate corrected QT (QTc) intervals were longer in patients who have appropriate therapy $(\mathrm{p}<0.01$ and $\mathrm{p}<0.001$, respectively.). QRS duration was similar between groups $(p=0.28)$. Number of patients with $T$ wave inversion was higher in the appropriate therapy group $(p=0.005)$ and TAaVR values were significantly associated with appropriate therapy $(\mathrm{p}<0.001)$. 
Table 1: Baseline clinical and demographic characteristics, laboratory parameters and medications of the study subjects.

\begin{tabular}{|l|l|l|l|}
\hline & Therapy (-) (n=27) & Therapy (+) (n=9) & P value \\
\hline Male (\%) & $74(20)$ & $66(6)$ & 0.66 \\
\hline Age (years) & $51 \pm 10.9$ & $49 \pm 8.3$ & 0.64 \\
\hline Diabetes, (\%) $\mathbf{n}$ & $33(9)$ & $11(1)$ & 0.19 \\
\hline Hypertension, (\%) $\mathbf{n}$ & $51(14)$ & $22(2)$ & 0.12 \\
\hline Atrial Fibrillation, (\%) $\mathbf{n}$ & $7(2)$ & $11(1)$ & 0.72 \\
\hline Glucose (mg/dL) & $99 \pm 18.0$ & $97 \pm 17.5$ & 0.58 \\
\hline Creatinine (mg/dL) & $0.87 \pm 0.23$ & $0.85 \pm 0.13$ & 0.85 \\
\hline Na (mEq/L) & $132 \pm 24$ & $137 \pm 2.1$ & 0.66 \\
\hline K (mEq/L) & $4.6 \pm 0.5$ & $4.4 \pm 0.7$ & 0.59 \\
\hline AST (mg/dL) & $25(11-49)$ & $30(16-65)$ & 0.50 \\
\hline ALT (mg/dL) & $24 \pm 13$ & $25 \pm 11$ & 0.85 \\
\hline LDL (mg/dL) & $117 \pm 42$ & $110 \pm 55$ & 0.75 \\
\hline HDL (mg/dL) & $39 \pm 13$ & $44 \pm 33$ & 0.64 \\
\hline Wight blood cell, $\mathbf{x 1 0 9 / L}$ & $8.1 \pm 1.8$ & $9.0 \pm 1.0$ & 0.30 \\
\hline Hemoglobin (mg/dL) & $13.0 \pm 1.7$ & $13.4 \pm 1.0$ & 0.57 \\
\hline Beta-blocker n (\%) & $85(23)$ & $88(8)$ & 0.78 \\
\hline ACEI-ARB, (\%) $\mathbf{n}$ & $85(23)$ & $55(5)$ & 0.08 \\
\hline Digoxin, (\%) $\mathbf{n}$ & $14(4)$ & $11(1)$ & 0.63 \\
\hline Spironolactone, (\%) $\mathbf{n}$ & $37(10)$ & $44(4)$ & 0.71 \\
\hline & & & \\
\hline
\end{tabular}

Na: Sodium; K: Potassium; AST: Aspartate aminotransferase; AST: Alanine transaminase; LDL: Low-density lipoprotein; HDL: High-density lipoprotein; ACEI: Angiotensin converting enzyme inhibitor; ARB: Angiotensin receptor blocker

Table 2: Echocardiographic and electrocardiographic results of the patients.

\begin{tabular}{|l|l|l|l|}
\hline & Therapy (-) (n=27) & Therapy (+) (n=9) & P value \\
\hline Ejection Fraction (\%) & $60 \pm 2.9$ & $62 \pm 4.5$ & 0.22 \\
\hline LVEDD (mm) & $45 \pm 4.8$ & $46 \pm 3.1$ & 0.44 \\
\hline LVESD (mm) & $30 \pm 4.1$ & $29 \pm 3.9$ & 0.36 \\
\hline Heart rate (bpm) & $73 \pm 11.3$ & $79 \pm 11.5$ & 0.19 \\
\hline QT interval (ms) & $378 \pm 17.8$ & $397 \pm 19.5$ & 0.01 \\
\hline QTc interval (ms) & $404 \pm 32.5$ & $437 \pm 55.5$ & $<0.001$ \\
\hline QRS interval (ms) & $94 \pm 11$ & $99 \pm 14$ & 0.28 \\
\hline T wave inversion, (\%)n & $22(6)$ & $77(7)$ & 0.005 \\
\hline TaVR & $-0,04 \pm 0.03$ & $-0,17 \pm 0.04$ & $<0.001$ \\
\hline
\end{tabular}

Data are represented as mean values $\pm S D$. LVEDD: Left Ventricle End Diastolic Diameter; LVESD: Left Ventricle End Systolic Diameter; mm: millimeters; bpm: beats per minute; TA$a V R, T$ wave amplitude in lead aVR.

\section{DISCUSSION}

Hypertrophic cardimyopathy is a genetic cardiovascular disease and characterized by increased wall thickness in which left ventricle of the heart due to subaortic stenosis without a certain cause. HCM is a common disease with a prevalence of one in 500 people of both genders $(1,2)$.

Myocardial ischemia, atrial fibrillation and heart failure risks are increased in patients with HCM. Atrial fibrillation is seen in $20 \%$ of patients and is the most common arrhythmia in HCM (1). Atrial fibrillation affects patients with HCM by increasing the risk of embolic stroke and heart failure. Those risks are increased in patients with left ventricular outflow obstruction. Aging and left atrial enlargement are associated with susceptibility to atrial fibrillation. Atrial fibrillation has not been explained by a particular genetic substrate or mitral regurgitation or left-ventricular outflow obstruction but there is no evidence about an independent cause of sudden death in atrial fibrillation (2).

In this study, it is found that the frequency of patients with more negative TAaVR was significantly higher in the therapy positive group than the therapy negative group $(\mathrm{p}<0.001)$. For this reason, T wave amplitude in lead aVR can be used to predict and evaluate appropriate of ICD therapy in HCM patients.

The importance of the lead aVR is usually neglected. However, ST elevation in lead aVR has been reported in left main coronary artery disease prediction (11). Furthermore, aVR is important in the evaluation of Brugada syndrome and it can be used for risk stratification (12).

In other respects, $\mathrm{T}$ wave amplitude in lead aVR can be used to evaluate the prognosis of diseases. It was demonstrated in dialysis patients that, positive TAaVR was related to all-cause mortality (13). In addition, a research by Tanaka et al. (14) reported that TAaVR was used for prediction of cardiovascular events in ischemic and nonischemic cardiomyopathy patients with ICD.

In the end of the study, we concluded that a higher TAaVR is associated with appropriate therapies in HCM patients who have ICD implants. Using simple ECG parameters, we may predict arrhythmic episodes before ICD implantation and an improvement of the medical antiarrhythmic therapy might be protective for HCM patients with ICD. 
Ethics Committee Approval: This study was approved by Scientific Researches Committee of Trakya University School of Medicine.

Informed Consent: Written informed consent was obtained from the participants of this study.

Conflict of Interest: The authors declared no conflict of interest.

Author contributions: Concept: BS, ÇG, PEH, CK, FMU. Design: BS, ÇG, PEH, CK, FMU. Supervision: BS, ÇG, PEH, CK, FMU. Resources: BS, ÇG, PEH, CK, FMU. Materials: BS, ÇG, PEH, CK, FMU. Data collection and/or processing: BS, ÇG, PEH, CK, FMU. Analysis and/or Interpretation: BS, ÇG, PEH, CK, FMU. Literature Search: BS, ÇG, PEH, CK, FMU. Writing Manuscript: BS, ÇG, PEH, CK, FMU. Critical Review: BS, ÇG, PEH, CK, FMU.

Financial disclosure: The authors declared that this study received no financial support.

Editor-in-chief's Note: Four of the authors of this article, Begüm Söyleyici, Pelinsu Elif Hünkar, Çağrı Girit and Cansu Kurt are members of the editorial board of Turkish Medical Student Journal. However, they did not take place in any stage on the editorial decision of the manuscript. The editors who evaluated this manuscript are from another institutions.

\section{REFERENCES}

1. Maroon BJ, Maroon MS. Hypertrophic cardiomyopathy. Lancet 2013; 81:242-55.

2. Liew AC, Vassiliou VS, Cooper R et al. Hypertrophic cardiomyopathy-past, present and future. J Clin Med 2017;6(12):118.

3. Spirito P, Autore C, Formisano F et al. Risk of sudden death and outcome in patients with hypertrophic cardiomyopathy with benign presentation and without risk factors. Am J Cardiol 2014;113:1550-5.

4. Veselka J, Zemanek D, Jahnlova D et al. Risk and causes of death in patients after alcohol septal ablation for hypertrophic obstructive cardiomyopathy. Can J Cardiol 2015;31:1245-51.

5. Maron BJ, Rowin EJ, Casey SA et al. Hypertrophic cardiomyopathy in adulthood associated with low cardiovascular mortality with contemporary management strategies. J Am Coll Cardiol 2015;65:1915-28.
6. Sears SF, Conti JB. Quality of life and psychological functioning of ICD patients. Heart 2002;87:488-93.

7. Moss AJ, Schuger C, Beck CA et al. Reduction in inappropriate therapy and mortality through ICD programming. N Engl J Med 2012;367:2275-83.

8. Özkartal T, Breitenstein A, Saguner AM et al. The subcutaneous implantable cardioverter defibrillator in daily clinical practice. Swiss Med Wkly 2017 9;147:w14518.

9. Konno T, Fujino N, Hayashi K et al. Differences in the diagnostic value of various criteria of negative $\mathrm{T}$ waves for hypertrophic cardiomyopathy based on a molecular genetic diagnosis. Clin Sci (Lond) 2007;112(11):577-82.

10. Tan SY, Engel G, Myers J et al. The prognostic value of T wave amplitude in lead aVR in males. Ann Noninvasive Electrocardiol 2008;13(2):113-9.

11. Kireyev D, Arkhipov MV, Zador ST et al. Clinical utility of aVR-The neglected electrocardiographic lead. Ann Noninvasive Electrocardiol 2010;15(2):175-80.

12. Babai Bigi MA, Aslani A, Shahrzad S. aVR sign as a risk factor for life-threatening arrhythmic events in patients with brugada syndrome. Heart Rhythm 2007;4(8):1009-12.

13. Matsukane A, Hayashi T, Tanaka Y et al. Usefulness of an upright T-wave in lead aVR for predicting the short-term prognosis of incident hemodialysis patients: a potential tool for screening high-risk hemodialysis patients. Cardiorenal Med 2015;5(4):267-77.

14. Tanaka Y, Konno T, Tamura Y et al. Impact of T wave amplitude in lead aVR on predicting cardiac events in ischemic and nonischemic cardiomyopathy patients with an implantable cardioverter defibrillator. Ann Noninvasive Electrocardiol 2017;22:1245-53. 